Appeared in British Journal for the Philosophy of Science 52 (2001) 539-573

\title{
Sets, Classes and Categories
}

by

\author{
F.A. Muller ${ }^{\dagger}$
}

Utrecht University

October 2000

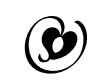

Summary

This paper, intended to be accessible for a general philosophical audience having only some fleeting acquaintance with set-theory and category-theory, concerns the philosophy of mathematics, specifically the bearing of category-theory on the foundations of mathematics. The author defends six claims. (I) A founding theory for category-theory based on the primitive concept of a set or a class is worthwile to pursue. (II) The extant set-theoretical founding theories for category-theory are conceptually flawed. (III) The conceptual distinction between a set and a class can be seen to be formally codified in Ackermann's axiomatisation of set-theory. (IV) A slight but significant deductive extension of Ackermann's theory of sets and classes founds Cantorian set-theory as well as category-theory and therefore can pass as a founding theory of the whole of mathematics. $(V)$ The extended theory does not suffer from the conceptual flaws of the extant set-theoretical founding theories. (VI) The extended theory is not only conceptually but also logically superior to the competing set-theories because its consistency can be proved on the basis of weaker assumptions than the consistency of the competition.
\end{abstract}

${ }^{\dagger}$ Department of the History and Foundations of Mathematics and Natural Science, Utrecht University, P.O. Box 80.000, 3508 TA Utrecht, The Netherlands; E-mail: f.a.muller@phys.uu.nl; homepage: http://phys.uu.nl/̌wwwgrnsl/\#muller 


\section{Contents}

1 Exordium 1

1.1 Motivation . . . . . . . . . . . . . . . . . 1

1.2 Overview ........................ 4

1.3 Terminology ..................... 4

2 Category-Theory versus Set-Theory 5

2.1 The Downfall of Set-Theory . . . . . . . . . . . . . 5

2.2 The Resurrection of Set-Theory . . . . . . . . . . . . . . . 8

2.3 Criticism of the Resurrection . . . . . . . . . . . . . . . . . 10

3 Sets and Classes $\quad 12$

3.1 Sets as Classes . . . . . . . . . . . . . . . . . . . . . 12

3.2 Requirements for a Founding Theory . . . . . . . . . . . . . 14

4 A Theory of Sets and Classes $\quad 16$

4.1 The Language of Sets and Classes . . . . . . . . . . . . . . 16

4.2 Axioms for Sets and Classes . . . . . . . . . . . . . . 17

4.3 Logical Respect . . . . . . . . . . . . . . . . . . . 23

4.4 The Lévy-Vaught Theorem . . . . . . . . . . . . . . . . . . . . 23

5 Conclusions and Reflections 25

5.1 Neo-Bourbakistan . . . . . . . . . . . . . . . . . . 25

5.2 Classes and Membership: Superfluous or Unavoidable? . . . . . 29

$\begin{array}{ll}\text { References } & 31\end{array}$ 


\section{Exordium}

\subsection{Motivation}

The Rise of Category-Theory. We take for granted the impact on and fertility of categorytheory for mathematics, logic and, more recently, mathematical physics. ${ }^{1}$ Readers of this journal have been served over the years one paper per decade on the subject-matter of category-theory: a critical voice by Mayberry [1977], who has vehemently rejected category-theory as the provider of foundations for the whole of mathematics; another critical voice by Bell [1981] who, at another place at another time, became a convert (Bell [1986]); and a "philosophical history" of the subject by McLarty [1990], who criticised Mayberry severely. The present paper is neither a response to nor prompted by the mentioned papers, although it will now and again refer to what is said therein. In the light of the pervasive use of category-theoretical methods in many branches of mathematics (think only of commutative diagrams) and of Lawvere's daring claim on the founding throne of mathematics on behalf of category-theory, comparisons to set-theory obtrude.

We do not subscribe to the view there ought to be a single occupant of the founding throne for mathematics. On the contrary, we submit a view that presumably is shared by most mathematicians because it explains the peaceful status quo - no founding battle is raging in mathematics as we speak. This view reads that more founding theories of mathematics increase our understanding of the nature of mathematical knowledge, just as the various axiomatisations of Euklidean geometry and quantum mechanics, say, increase our understanding of these theories. We therefore turn to the only two theories that can legitimately lay a claim on being a founding theory of the whole of mathematics, for that will be the subject-matter of the present paper. In order to state the aim of this paper more precisely, we need a few more introductory sentences.

Category-theory axiomatises the notions of an object (about which next to nothing is assumed) and of an arrow and arrow-composition, just like set-theory axiomatises the notions of a set and the membership-relation; we denote their respective 1st-order formal languages by $\mathcal{L}_{\epsilon}$ and $\mathcal{L}_{\uparrow}$ (see Fraenkel et al. $[1973$, h. 1 ).

Axioms can be seen as extra-logical deduction-rules and therefore tell us, together with the formation-rules of the language, how the primitive concepts are permitted to be used; by their use concepts acquire meaning (Wittgenstein). The standard axiomatisation of set-theory is what Von Neumann [1925; 1928; 1961, p. 321, 348] baptised ZermeloFraenkel set-theory (ZFC), although a slight reformulation of Von Neumann's own functiontheory, called Cantor-Von Neumann set-theory $(\mathrm{CVN})$, is the superior legislator of what Hilbert has baptised 'Cantor's Paradise'. ${ }^{2}$

\footnotetext{
${ }^{1}$ For an impossible overview of the entire field of mathematics, category-theory included, see magister Saunders Mac Lane's [1986, pp. 386-408]; see further J.L. Bell's philosophically oriented papers on the subject-matter [1981; 1982; 1986]. The most accessible introduction to date remains Goldblatt [1979], which includes a category-theoretical approach to Logic.

${ }^{2}$ Von Neumann's theory became known among set-theoreticians after Paul Bernays moulded it into a
} 
Lawvere [1966] proposed CAT, the category of all categories (save itself), as the domain of discourse of mathematics, and embarked on the endeavour to axiomatise CAT directly:

In the mathematical development of recent decades one sees clearly the rise of the conviction that the relevant properties of mathematical objects are those which can be stated in terms of their abstract structure rather than in terms of the elements which the objects were thought to be made of. The question thus naturally arises whether one can give a foundation for mathematics which expresses wholeheartedly this conviction concerning what mathematics is about, and in particular in which classes and membership in classes do not play any rôle.

Founding father Mac Lane [1968] effectively sketched how to translate $\mathcal{L}_{\uparrow}$ into $\mathcal{L}_{\epsilon}$ and extended ZFC to a more encompassing set-theory in order to found category-theory (see below). More than a quarter of a century later, Mac Lane subscribes to the following view [1994, p.30]:

With recent increasing use, the question of proper foundations has come to the fore. Here experts are still not in agreement; our present assumption of 'one universe' is an adequate stopgap, not a forecast of the future.

In short, for category-theory there still is, half a century after its birth, no universally accepted axiomatisation - a remarkable state of affairs that calls for an explanation. All putative axiomatisations of category-theory stand in marked contrast to the simple axioms of ZFC in that the afore-mentioned can be understood if and only if comparatively sophisticated knowledge of category-theory is available.

Challenges. The situation of having two theories that found mathematics presents proponents of each theory with a challenge. In order to remain on the founding throne of mathematics, set-theory faces the challenge to found category-theory. If some set-theory founds category-theory, then all entities in the domain of discourse of category-theory are construed as entities in the domain of discourse of set-theory, i.e. as sets. Since besides category-theory all other branches of mathematics are or can unproblematically be founded on set-theory, the only challenge that faces set-theory is to found category-theory. Category-theory faces the converse challenge. This is also the only challenge that faces category-theory, because if set-theory is reduced to a branch of category-theory, then all results of the founding labour in the past on the basis of set-theory (notably the reduction of all other branches of mathematics to set-theory) can very probably be transported to category-theory without much ado.

But why should we face these challenges at all? What is so interesting about them? Claim I reads that it is worthwile to pursue set-theoretical foundations for category-theory. So to answer this question is to argue in favour of Claim I. We shall next mention five reasons; they provide the motivation of the present paper.

logicist class-theory; the moulded theory is known under the name of 'Von Neumann-Bernays set-theory', or even 'Bernays-Gödel set-theory'. For all of this, see Muller [1998, pp. 411-477] and [2000]. 
The first, perhaps trivial reason is that the sheer presence of these intellectual challenges is sufficient motivation to face them - think of the answer George Mallory provided to the question why he wanted to climb Mount Everest.

Secondly, when we take it for granted there are various epistemic and ontic considerations that motivate the hunt for a founding theory of mathematics, then, in the light of the founding claims that both set-theory and category-theory lay, the project to found settheory on category-theory and the converse project are eo ipso motivated. Suppose you are a Platonist, who holds there is some realm of existence beyond space-time populated by the entities of mathematics; they are causally inept but somehow epistemically accessible for us. A founding theory then gives you a clear idea what the population of this Platonic realm is. Suppose you are a Nominalist, who holds there is no such thing as the Platonic realm; the names and descriptions in the languages of mathematical theories do not refer to some extra-linguistic reality; their meaning derives from their use. A founding theory then gives you a clear idea what the ultimate primitive concepts of mathematics are and the 'semantic rules' govering their rigourous use. So in both cases a founding theory clarifies something about mathematics and is therefore of interest to the philosophy of mathematics.

Thirdly, it is intrinsically interesting to know how these theories of mathematics compare, irrespective of their foundational capacities, because of the generality of their pivotal concepts and their widespread use in mathematics. Such knowledge constitutes knowledge about the conceptual workings of mathematics.

Fourthly, to understand how category-theory relates to set-theory is to understand how a novel mathematical theory relates to the mathematical tradition. Mathematical knowledge, independent of your favourite philosophical view on it, is undeniably growing and it is growing rapidly in ever more directions. When we want to know how the (comparatively) novel category-theory relates to the extant body of mathematical knowledge, if only because we want to be convinced we can still speak of a single body of mathematical knowledge, then we certainly are satisfied when it is demonstrated that settheory founds category-theory, because the last-mentioned theory poses the only threat to the afore-mentioned theory as a founding theory. If it turns out that the standard axioms of set-theory need to be strengthened to achieve this, then we have the perfect reason to conclude that category-theory is not only - to remain in the metaphor -, an extension in the width of the body of mathematical knowledge, but also an extension in depth.

Fifthly, we mention Bourbaki's structural architecture of mathematics and the outand-out structural nature of category-theory. Bourbaki erected his structural architecture of mathematics, which is in spirit category-theoretical with its uncompromising emphasis on form and structure, on the basis of set-theory. ${ }^{3}$ When we now realise that categorytheory was more-or-less created by Bourbaki ${ }^{4}$, and that Bourbaki's exposition of his ar-

\footnotetext{
${ }^{3}$ Bourbaki [1949; 1950]. See Muller [1998, pp. 21-126] for an overview and evalutation.

${ }^{4}$ Eilenberg and Grothendieck belonged to Bourbaki; Mac Lane did not, but he worked in Chicago and we know for a fact that Bourbaki resided in Nancago - concatenation of Nancy (France) and Chicago (United
} 
chitecture, in spite of its set-theoretical foundations, frequently deploys typical categorytheoretical means (natural transformations, commutative diagrams), we expect categorytheory to fit in seamlessly. So the fifth reason to pursue set-theoretical foundations for category-theory is to verify this expectation and thus to verify that a category is 'just another' species of Bourbakian structure - yet such an important one that it ought to be counted among Bourbaki's 'mother structures'.

\subsection{Overview}

We commence in the next Section by quickly rehearsing the reasons why standard settheory is unable to found category-theory $(\$ 2.1)$ and give an overview from the bird's eye point of view of new, stronger set-theories whose promulgators claim they found category-theory ( $\$ 2.2)$. We claim however that all these stronger set-theories are conceptually flawed and that every other pure set-theory will be flawed too (Claim II, §2.3). In $\S 3$ we briefly rehearse the differences and agreements between the concept of a set and of a class (§3.1) and list six requirements that a certain kind of founding theory for mathematics has to meet (§3.2).

In $\S 4$ we re-interpret and strengthen Ackermann's set-theory of 1956, which results in a theory we abbreviate as ARC (Sections 4.1 and 4.2). Ackermann intended his theory to be a thoroughly Cantorian set-theory, in some respects improving upon ZFC (such as recognising and admitting 'absolute-infinite' wholes). But we re-interpret it as a theory of sets and classes (Claim III: this is possible), which means we shall qualitatively extend the realm of pure set-theory. We further discuss a few useful theorems of ARC $(\S 4.4)$ and a few meta-theorems, e.g. its relation to ZFC, in order to make ARC, besides conceputally, logically respectable too $(\S 4.3)$.

We next sketch how the revision of Bourbakistan we propose can be founded on ARC, and how ARC encompasses both Cantorian set-theory and category-theory ( 55.1 ); thence it follows that ARC founds mathematics (Claim IV) without suffering from the flaws of the set-theoretic competition (Claim V), and without the need for so strong an axiom as the competition believes to be inevitable to prove its consistency (Claim VI). We end by arguing that although the structures of some branches of mathematics are more conveniently handled by means of category-theory, this does not imply the inadequacy of theory of sets and classes as a founding theory (§5.2).

Before we embark on our endeavour, we need to fix a few terminological conventions and to recall a few results.

\subsection{Terminology}

For theory $\mathrm{Th}_{2}$ formulated in language $\mathcal{L}_{2}$ to found $\mathrm{Th}_{1}$ formulated in language $\mathcal{L}_{1}$, we say it is necessary and (until further notice) sufficient to interpret $\mathrm{Th}_{1}$ in $\mathrm{Th}_{2}$ in Tarski's

States of America). 
sense, i.e. to translate $\mathcal{L}_{1}$ into $\mathcal{L}_{2}$ and to prove all translated theorems of $T h_{1}$ in $T h_{2}$. When both theories have the same deductive apparatus, as in the present case of set-theory and category-theory, namely 1st-order classical predicate logic, it suffices to prove the translated axioms of $T_{1}$ in $T h_{2}$. Now, to found mathematics on theory $T$ means to found all theories of mathematics on $\mathrm{T}$. Since the absurdity $(\perp)$ is a translation-invariant, the proof of an interpretation is also a relative consistency-proof: if $T h_{2}$ interprets $T h_{1}$, then $\mathrm{Th}_{1}$ is consistent relative to $T h_{2}$. So if theory $\mathrm{T}$ founds mathematics, then all mathematical theories are consistent if $\mathrm{T}$ is. We notice that this notion of 'foundation' is rather strong: it is both 'logical' and 'ontological'.

Theory $T h_{2}$ deductively extends theory $T h_{1}$ iff language $\mathcal{L}_{2}$ inlcudes language $\mathcal{L}_{1}$ and $T h_{2}$ includes $T h_{1}$; the deductive extension $T h_{2}$ is conservative over $T h_{1}$ iff all theorems of $T h_{2}$ in the language $\mathcal{L}_{1}$ are already theorems of $T h_{1}$, i.e. $\mathcal{L}_{1} \cap T h_{2}=T h_{1}$; and it is progressive iff it is not conservative. One easily proves that if $T h_{2}$ is a conservative extension of $T h_{1}$, or vice versa, then $T h_{2}$ and $T h_{1}$ are equiconsistent.

Further we use the symbol $\equiv$ for term-definition (left the definiendum and right the definiens) and $\Longleftrightarrow$ for sentence-definition (idem dito). Throughout the paper we use 'informal rigour' (Kreisel).

\section{Category-Theory versus Set-Theory}

\subsection{The Downfall of Set-Theory}

Category-Theoretical Foundations of Set-Theory. Although founding set-theory on categorytheory is not the topic of this paper, we want to devote a few sentences to it nonetheless for the sake of overview. The enterprise to give simple definitions of a set and the membership-relation in terms of objects, arrows and their composition ran initially into serious difficulties, as Bell [1981, p. 355] reported; they have been overcome, because in topos-theory, a prominent branch of category-theory, a route is walked similar to how Von Neumann [1925; 1961, p. 38] defined the membership-relation in terms of characteristic functions, namely as a 'sub-object classifier'; see Osius [1974] and Bell [1982, p. 306 ff.]. Joyal \& Moerdijk [1995] explore another avenue to obtain sets and the membershiprelation. By observing how the cumulative hierarchy arises, namely by making powersets, union-sets and replacement-sets, they arrive at a category they call a Zermelo-Fraenkel algebra; they succeed, first, in providing a a binary relation which is algebraically indistinguishable from our familiar $\in$ and, secondly, in constructing, as they call it, "simulacrum ordinal numbers". This leads to a proof of all the axioms of ZFC (ibid. p.18, 29). All research in set-theory can now be construed as the study of the Zermelo-Fraenkel algebra. One cannot qualify these reductions as natural, simple or elegant. But certainlyl they are possible. And now we turn to the main topic of this paper.

Set-Theoretical Definitions of the Basic Category-Theoretical Concepts. We present definitions in $\mathcal{L}_{\epsilon}$ of the primitive concepts of category-theory and of a few central defined ones. 
To begin with, an object simply is a set.

The standard definition in $\mathcal{L}_{\epsilon}$ of function $f$ from set $D$ to $C$, due to Hausdorff, is to take $f$ as a relation between the members of $D$ and $C$, i.e. a subset of the Cartesian product-set $D \times C$, that relates to every member in $D$ exactly one member of $C$. This makes $f$ a particular kind of set of ordered pairs $\langle x, f(x)\rangle \in D \times C$. This set $f \in \wp(D \times C)$ determines its domain $D_{f}\left(=D\right.$ whenever $D$ is the given domain) and its range $R_{f}$, but not its co-domain: $C \supseteq R_{f}$. We now define an arrow as an ordered pair $\langle f, C\rangle$ of a function $f$ and a given co-domain $C$; in this fashion an arrow trivially determines its co-domain, as it should. This reveals that the arrow-notation $f: D \rightarrow C$ is tailor-made for arrows but a misfit for functions. Ordered pairs, and polytuples generally, can be defined in a variety of manners (Wiener, Kuratowski, Haussdorf, Skolem, Quine, Kitcher); any choice will do.

Let $\{D \rightarrow C\} \subset \wp(D \times C)$ denote the set of all arrows $\langle f, C\rangle$ with domain $D$ and co-domain $C$. The identity-arrow on $D$ now simply is $\left\langle\operatorname{id}_{D}, D\right\rangle$, where $i_{D}$ is the set of ordered pairs $\langle x, x\rangle$ for all $x \in D$; this 'diagonal-set' of $D$ also qualifies as a function, the identity-function on $D$. The definition of composition of arrows is essentially the same as the definition of composition of functions: $h: X \rightarrow Z$ is the arrow-composition of $f: X \rightarrow Y$ and $g: Y \rightarrow Z$, denoted by $h=g \circ f$, iff

$$
\forall x \in X, \exists y \in Y(f(x)=y \wedge g(y)=h(x)) .
$$

In every set-theory one easily proves that arrow-composition is associative (an axiom in category-theory), and that $\operatorname{id}_{D}: D \rightarrow D$ meets the defining conditions of an identityarrow on $D$ in category-theory: $f \circ \mathrm{id}_{D}=f$ for all $f: X \rightarrow D$ and $\operatorname{id}_{D} \circ g=g$ for all $g: D \rightarrow Y$.

Since all concepts in category-theory are defined in terms of its primitives (object, arrow and composition), which we now have translated in $\mathcal{L}_{\epsilon}$, all defined concepts of category-theory, e.g. functor, functor-category, natural transformation, topos (a particular kind of category), allegory, can be translated in $\mathcal{L}_{\epsilon}$. We shall nonetheless indicate how some of the major defined notions of category-theory are defined in $\mathcal{L}_{\epsilon}$.

We define the set $\vec{B}$ of exactly the arrows in $B$, i.e. whose domain and co-domain are members of set $B$ :

$$
\vec{B} \equiv\{\langle f, Y\rangle \in\{X \rightarrow Y\} \mid X, Y \in B\}=\cup_{X, Y \in B}\{X \rightarrow Y\} .
$$

A category $C$ we provisionally define as a particular species of Bourbakian set-structure, namely as an ordered triple consisting of: a non-empty set $B$ (which harbours the objects); a set $\mathcal{A}(B) \subseteq \vec{B}$ of arrows in $C$ which contains all identity-arrows in $\vec{B}$; and the composition-operation $\circ$ between arrows in $\mathcal{A}(B)$ such that this set is closed under it:

$$
C \equiv\langle B, \mathcal{A}(B), \circ\rangle \text {. }
$$

Composition is a function (not an arrow) from $\mathcal{A}(B) \times \mathcal{A}(B)$ to $\mathcal{A}(B)$. In Bourbakian terminology: set $B$ is the 'Base-set' of structure (3) and $\mathcal{A}(B)$ and $\circ$ are 'echelon-sets'. ${ }^{5}$ Hence

\footnotetext{
${ }^{5}$ See Corry [1992] for the historical career of Bourbaki's conception of structure; see Muller [1998, pp. 106115] for an accessible discussion of Bourbaki's rigourous definition and for numerous illustrations.
} 
a category is just an associative arrow-composition magma (Bourbaki calls a set equipped with a single operation a 'magma' — volcanic terminology referring to hot material underground?).

A functor $F$ from category $X=\langle X, \mathcal{A}(X)$, o $\rangle$ with base-set $X$ to category $Y=\left\langle Y, \mathcal{A}(Y), \circ^{\prime}\right\rangle$ with base-set $Y$, denoted by $F: X \rightarrow Y$, we define as an ordered pair of arrows:

$$
F \equiv\left\langle F_{\mathrm{obj}}, F_{\mathrm{arr}}\right\rangle, \quad \text { were } \quad F_{\mathrm{obj}} \equiv\left\langle f_{\mathrm{obj}}, Y\right\rangle \quad \text { and } \quad F_{\text {arr }} \equiv\left\langle f_{\mathrm{arr}}, \mathcal{A}(Y)\right\rangle,
$$

where function $f_{\text {obj }}$ sends objects in $X$ to objects in $Y$, and where function $f_{\text {arr, }}$ which sends arrows of $\boldsymbol{X}$ to arrows of $\boldsymbol{Y}$, is such that it preserves composition and sends identityarrows to identity arrows, i.e. $f_{\text {arr }}$ is an algebraic morphism with regard to arrow-composition (it preserves the composition-structure). The set of all functors from $X$ to $Y$, we denote by Func $(X, Y)$, then is related to $X$ and $Y$ as follows:

$$
\operatorname{Func}(X, Y) \equiv\{F \in\{X \rightarrow Y\} \times\{\mathcal{A}(X) \rightarrow \mathcal{A}(Y)\}\}
$$

A functor-category of $\boldsymbol{X}$ to $\boldsymbol{Y}$, denoted by $\boldsymbol{Y}^{\boldsymbol{X}}$, is a category whose objects are all functors from $X$ to $Y$ and whose arrows send functors to other functors:

$$
\boldsymbol{Y}^{\boldsymbol{X}} \equiv\langle\operatorname{Func}(\boldsymbol{X}, \boldsymbol{Y}), \mathcal{A}(\operatorname{Func}(\boldsymbol{X}, \boldsymbol{Y})), \circ\rangle \text {. }
$$

The natural transformations are among the arrows of this functor-category.

There is nothing wrong with these definitions in $\mathcal{L}_{\epsilon}$. There is however something wrong with the axioms of standard set-theory, ZFC, or CVN for that matter, because on the basis of these axioms one proves that virtually all category-theoretical objects, when defined as sets, turn out not to exist! We next rehearse a few examples to illustrate this.

Non-Existent Sets. Let $\mathbf{V}$ be domain of discourse of set-theory; as the set of all sets it does not exist according to ZFC, but as the ultimate set of all Cantor-sets, it does exist according to CVN. ${ }^{6}$ Categories whose base-set of objects exists as a set according to ZFC are called 'small' (ZFC-sets; they are legitimate members of $\mathbf{V}$ according to CVN); and those who demonstrably can be construed as subsets (but not as members) of $\mathbf{V}$ are called 'large' (the absolute-infinite sets of CVN). ${ }^{7}$ Categories which are neither small nor large lie beyond the mentioned set-theories.

For the sake of concreteness and future reference, we mention a few familiar categories: Set has as its objects all sets (members of $\mathbf{V}$ ) and as its arrows all functions between sets (the co-domains coincide with the ranges); $\mathrm{Cl}$ s has as its objects all subsets of $\mathbf{V}$ and as its arrows all functions between these; Grp has as its objects all group structures, i.e.

\footnotetext{
${ }^{6}$ An ultimate set is a set which is not a member of some other set - in CVN they are all equinumerous to V according to Von Neumann's Axiom of Limitation; a Cantor-set is a well-founded, increasible set which has a unique ordinal number when ordered well, and a unique cardinal number, where increasible means: included in a set amplinumerous to it. Theorem: a set is a Cantor-set iff it is not ultimate. See Muller [1998, pp. 411-475] for the why \& how of these concepts and (proofs of) similar theorems.

${ }^{7}$ These definitions were introduced by Mac Lane [1994, p. 23] for the sake of comparing category-theory to set-theory.
} 
quadruples of the form $\left\langle G, \diamond,(\cdot)^{\text {inv }}, 1\right\rangle$ obeying the set-theoretical predicate 'is a group', and has as its arrows all group-homomorphisms; Top has as its objects all topological structures, i.e. quadruples of the form $\langle S, T(S), \cup, \cap\rangle$, where $T(S)$ is the usual topological subset familly of set $S$ obeying the Hausdorff axioms, and has as its arrows all continuous mappings; Man has as its objects all differentiable manifolds and as its arrows all smooth maps; Cat is the category of all small categories, which are its objects, and the functors between them are its arrows. These categories are then defined in $\mathcal{L}_{\epsilon}$ as follows:

$$
\begin{aligned}
\text { Set } & \equiv\langle\mathbf{V}, \overrightarrow{\mathbf{V}}, \circ\rangle, \\
\text { Cls } & \equiv\langle\wp \mathbf{V}, \overrightarrow{\wp \mathbf{V}}, \circ\rangle, \\
\text { Grp } & \equiv\langle G, \operatorname{Hom}(G), \circ\rangle, \\
\text { Top } & \equiv\langle\boldsymbol{T}, \operatorname{Cont}(T), \circ\rangle, \\
\text { Man } & \equiv\langle M, \operatorname{Smooth}(M), \circ\rangle, \\
\text { Cat } & \equiv\left\langle C, \cup_{X, Y \in C} \operatorname{Func}(X, Y), \circ\right\rangle .
\end{aligned}
$$

These categories are large: their base-sets exist in CVN as ultimate subsets of $\mathbf{V}$, but neither the sets of their arrows nor the category structures (7) themselves exist (as ultimate sets). For example, on the basis of the Kuratowksi-definition of an ordered pair, Set is a member of a set a number of power-sets of $\mathbf{V}$ upward. From the definitions of functor and functor-category we see that all these, when starting from the large categories just mentioned, e.g. to obtain the functor-categories $S e t^{G r p}$ and Set ${ }^{T o p}$, do not exist either. Hence it is generally held that standard set-theories, notably ZFC and CVN, cannot provide foundations for the whole of mathematics. ${ }^{8}$

Conclusion: if one pursues set-theoretical foundations of category-theory, then the axioms of ZFC or CVN need to be strengthened. We next take a quick look at four extant manners of how to achieve this; they are all four formally distinct but some of them are very similar.

\subsection{The Resurrection of Set-Theory}

Mac Lane [1969; 1994, p.23] postulated inside the domain of discourse of ZFC, which is $\mathbf{V}$, a copy of sorts of $\mathbf{V}$ that plays the rôle of a domain of discourse for ZFC just as good as $\mathbf{V}$ itself. This move suffices to found category-theory set-theoretically, Mac Lane propounded and still propounds in his [1994, pp. 21-23].

Definition: a MacLane universe is (U1) a transitive set which (U2) contains the set of all finite ordinals $(\mathbb{N}),(\mathrm{U} 3)$ is closed under the formation of power-sets and union-sets, and (U4) contains the ranges of all functions whose domain it contains and whose range it includes, i.e. it is closed under the formation of replacement-sets. Observe that $\mathbf{V}$ does not qualify as a MacLane universe because it is not a set. The Universe Axiom (U) states that

\footnotetext{
${ }^{8}$ Mac Lane [1994, pp. 23-24], McLarty [1990, p. 366].
} 
there exists a MacLane universe. Call it U; theory ZFCU is the deductive extension of ZFC with $U$. Set $U$ by definition cannot be reached from inside $\mathbf{V}$ by making power-sets, unionsets and replacement-sets; $U$ has the first non-denumerable inaccessible cardinality ( $\# U=$ $\mathfrak{i}_{1}$, when $\left.\mathfrak{i}_{0} \equiv \aleph_{0}\right)$. It ought to be clear from the definition of a MacLane universe, whose conjuncts are essentially the Axiom of Infinity (U2), the Power and the Union Axiom (U3) and the Axiom of Replacement (U4), that $U$ provides us with a transitive model $\langle U, \in\rangle$ of ZFC (and of a variety of weaker set-theories for that matter). If $U$ could be derived from ZFC, then ZFC would thus contain a model of itself, which means, according to the Model Existence Lemma, that ZFC proves its own consistency; but this is impossible due to Gödel's Consistency Theorem, unless ZFC is inconsistent. Hence the consistency of ZFCU cannot be proved relative to ZFC. In order to prove the consistency of ZFCU one needs to axiomatically add two strongly non-denumerable inaccessible cardinals to $\mathbf{V}$ of ZFC ( $i_{1}$ and $\left.i_{2}\right)$, which results in $\mathrm{ZFC}^{++}$, say.

Mac Lane now re-defines a set to be small iff it is a member of $U$, otherwise large. Mac Lane [1969, p. 195]:

Our intention is that the small sets can serve as the objects of Mathematics, while the other sets, not necessarily small, may be used to describe the various categories and functor-categories of these Mathematical objects.

Mac Lane further illustrated how to capture the various other notions of category-theory in set-theoretical terms. ${ }^{9}$

Proposals similair to Mac Lane's are submitted by Feferman [1969], based on reflection principles and a primitive 'set of small sets' [(ibid., p. 218] (see also Kreisel in Mac Lane's[1969, pp. 201-247] for elucidating comments). Just as ZFCU, this theory needs a meta-theory with two inaccessibles to prove its consistency. And mutatis mutandis for Cantor-Von Neumann set-theory (CVN) when we throw in an inaccessible. ${ }^{10}$ Finally, Feferman [1977, pp.154-155] reports how Grothendieck once proposed a set-theory that does not have a single set-universe, as Mac Lane's ZFCU has, but for every category a different setuniverse (itself a set), such that every set-universe is contained in the next one: $U_{0} \in U_{1} \in$ $U_{2} \in \cdots$. This leads to a denumerable number of inaccessible cardinal numbers $\mathfrak{i}_{j}(j \in \mathbb{N})$ being added to ZFC, leading to $\mathrm{ZFC}^{\omega}$. Its consistency can only be proved in ZFC plus more inaccessible cardinal numbers than there are natural numbers.

So can we conclude there are no troubles anymore in Cantor's Paradise? Has settheory risen from its ashes, stronger than ever, and successfully founded category-theory? In the next Section we argue in favour of a denial.

\footnotetext{
${ }^{9}$ For further foundational discussion by Mac Lane, see his [1969; 1986, pp. 386-406; 1994, pp. 21-23].

${ }^{10}$ There are other, even more powerful theories of sets (and classes) than those just presented that are able to accommodate category-theory, but they are contrived and appalingly ad hoc: they lack any motivation and are still born. An example is the ga-ga theory called ST 2 , cf. Fraenkel [1973, pp. 142-143].
} 


\subsection{Criticism of the Resurrection}

We level two objections against the attempts outlined in the previous Section to found category-theory set-theoretically, which apply to all four of them. The objections are supposed to ground Claim II that all extant set-theoretical foundations are, no matter how impeccable logically, flawed conceptually.

First Objection: Superabundancy. Bourbaki defines a mathematical structure, $\mathfrak{U}$ say, as a polytuple of sets: the first occupants are plain sets, so-called base-sets, and the other occupants, e.g. relations, functions, mappings, operators, operations, subset families, sets of operators, sets of functions and what have you, are constructed by set-theoretical means from the base-sets and are called echelon-sets. One now can define the niche of $\mathfrak{U}$, denoted by $\mathrm{Ni}(\mathfrak{U})$, as the set which includes and contains all base-sets and echelon-sets of $\mathfrak{U}$; see Muller [1998, p. 114]. Bourbaki characterises branches of mathematics by the structures they study; then $\mathrm{Ni}(\mathfrak{U})$ can be conceived as the domain of discourse of the branch of mathematics characterised by $\mathfrak{U}$. If the structure $\mathfrak{U}$ is an $n$-tuple, then it is a member of the following $n$-fold Cartesian product-set: $\mathrm{Ni}(\mathfrak{U})^{n}$. For large categories, the base-set of objects is not a member of $\mathbf{V}$ but a subset of $\mathbf{V}$; for categories which are neither small nor large, the base-set simply does not exist. Then neither do the echelon-sets exist nor does the niche exist. However, if $\mathbf{V}$ exists and we can apply all the set-theoretical operations on $\mathbf{V}$ an arbitrary but finite number of times without blasting ourselves out of the domain of discourse of the theory that provides us with such a license, then every category will be a member of some 'set' that we can construct out of $\mathbf{V}$. We briefly elaborate.

Let us call $n \in \mathbb{N}$ the power of set $Y$ over $X$ iff $X \in \wp^{n} Y$. If some theory of sets permits the existence of domains of sufficiently large but finite power over $\mathbf{V}$, and permits the usual set-theoretical operations upon its members, then category-theory can be founded on this theory provided the objects and arrows are members of some set of some finite power over V. From this perspective, Bourbaki 'limited' his attention to a domain of power 0 over $\mathbf{V}$. We must generalise from 0 to arbitrary natural numbers. Notice that 'union-sets' are then also available: $\bigcup \wp^{n+1} \mathbf{V}=\wp^{n} \mathbf{V}$. For a member $X \in \wp^{n} \mathbf{V}$ we then have that $\wp X \in \wp^{n+1} \mathbf{V}, \cup X \in \wp^{n-1} \mathbf{V}$; and if $Y \in \wp^{m} \mathbf{V}$, then $\langle X, Y\rangle \in \wp^{2}\left(\wp^{n} \mathbf{V} \cup \wp^{m} \mathbf{V}\right)$ and $X \times Y \in \wp^{3}\left(\wp^{n} \mathbf{V} \cup \wp^{m} \mathbf{V}\right)$, when one adopts Kuratowski's definition of an ordered pair.

The conclusion of this brief informal analysis coincides partly with an assertion of Fraenkel, Bar-Hillel, Lévy and Van Dalen [1973, p. 143]:

Category Theory involves only objects which are members of the classes $\mathbf{V}, \wp \mathbf{V}$, $\wp \wp \mathbf{V}, \ldots, \wp^{n} \mathbf{V}$, where $\mathbf{V}$ is the class of all sets and $n$ is some fixed finite number.

This brings us to our first objection against the four attempts to found category-theory set-theoretically: they are not just $a$ bit abundant, they are mindboggingly, flabbergastingly abundant. In all four approaches the inaccessible cardinal $\mathfrak{i}_{1}$ exists and therefore we have an unimaginably transfinitude of ever increasing cumulation sets beyond $\Psi\left(\mathfrak{i}_{1}\right)$, 
where $\alpha \mapsto \mathbf{\Psi}(\alpha)$ is the set-cumulator ( $\alpha \in \Omega$, the ultimate set of all ordinals; the range of $\Psi$ is the cumulative hierarchy). Comparing the size of $\Psi\left(\mathfrak{i}_{1}\right)$ to the size of $\Psi\left(\mathfrak{i}_{1}+\omega^{\omega}\right)$, say, does not even begin to compare as a fire-cracker to a zillion Hydrogen bombs, say. We have to realise that $\Psi\left(\mathfrak{i}_{1}+\omega^{\omega}\right)$ only is a drop in an unlimited universe of sets still to come: $\Psi\left(\mathfrak{i}_{1}+\boldsymbol{\aleph}_{\omega}\right), \mathbf{\Psi}\left(\mathfrak{i}_{1}^{\omega}\right)$, etc. It keeps on increasing indefinitely. The gigantic universe that Feferman creates with ZFCS, that Mac Lane creates with ZFCU, we created with CVN $^{+}$ and Grothendieck with $\mathrm{ZFC}^{\omega}$, is so ridiculously large in comparison to what we actually need to found category-theory, that is unbelievable it has even been considered seriously. What is needed and sufficient is 'only' a denumerable sequence of increasing cumulation sets $\Psi\left(\mathfrak{i}_{1}+1\right)$ for $\wp \mathbf{V}, \mathbf{\Psi}\left(\mathfrak{i}_{1}+2\right)$ for $\wp^{2} \mathbf{V}$, until $\mathbf{\Psi}\left(\mathfrak{i}_{1}+\omega\right)$, but certainly no more.

In the light of the previous paragraph, Grothendieck's set-theory ZFC ${ }^{\omega}$, not with 1 or 1,000 or $1,000^{1,000}$ but with a denumerably transfinite number $\omega$ of inaccessibles in $\mathbf{V}$, simply is beyond perplexity.

If these proposals are so exessively comprehensive as this author says they are, then why have they been seriously considered in the first place? The answer is simple: because it is generally held that if you want to found category-theory on a set-theory of sorts, then there is no other way but to throw in inaccessibles. We shall presently see there is another way. But first we turn our attention to a second objection.

Second Objection: Insufficiency. In the three set-theories we do not only have far too much, but what we have is, paradoxically, not sufficient. Let us now denote by $\mathbf{V}^{+}$the domain of discourse of either theory ZFCU (Mac Lane), ZFC* (Feferman) or CVN ${ }^{+}$. When a category-theoretician talks about Set, he means all sets, i.e. all sets available in the domain of discourse of mathematics, which has now become $\mathbf{V}^{+}$, not $\mathbf{V} \subset \mathbf{V}^{+}$, because old $\mathbf{V}$ comprises less than a thee spoon of the sets in the indefinitely expanding cosmos $\mathbf{V}^{+}$. When a category-theoretician hears there are sets available of cardinality $\mathfrak{i}_{1}, 2^{\mathfrak{i}_{1}}, \mathfrak{i}_{1}^{\omega}, \boldsymbol{\aleph}_{\mathfrak{i}_{1}}$, etc., he means to include these too in Set, because these are also sets according to these stronger set-theories, not mathematical objects distinct from sets. But $\mathbf{V}^{+}$cannot in turn be the base-set of the category structure Set because it is not a set. A functor-category such as Set $^{G r p}$ lies manifestly beyond $\mathbf{V}^{+}$. All arguments demonstrating that category-theory blasts out of the confines of $\mathbf{V}$ can now be repeated with $\mathbf{V}^{+}$replacing $\mathbf{V}$. Our star-witness is founding father Mac Lane [1994, p. 24], who made the following confession concerning his own attempt (ZFCU):

Our foundation by means of one Universe does provide, within set-theory, an accurate way of discussing the category of all small sets and all small groups, but it does not provide sets to represent certain meta-categories [= categories outside $\mathrm{V},-\mathrm{FAM}$ ] such as the meta-category of all sets or that of all groups.

Any stipulation to the effect that the category-theoretician is only allowed to grab at some fixed set whereas outside this set there are more sets, so that he is not permitted to grab at all of them, is artificial and barks at his explicit intentions. The categorytheoretician has every right to refuse to dance to the cardinality tunes the set-theoretician 
whistles. Category-theory is about form \& structure, irrespective of how much \& how many; it 'only' wants to have everything which is available. The category-theoretician means all sets when he makes the category Set of all sets, period. Set-theories which cannot accommodate this are flawed. Even Grothendieck's stultifying theory ZFC ${ }^{\omega}$ is conceptually inadequate in this respect.

We conclude that Categoristan defeats the strongly inaccessible set-troopers with exactly the same weapons as it earlier defeated ZFC and CVN. No matter how many strongly inaccessible cardinals one mobilises against category-theory, certain defeat will await all such attempts. Not a single pure set-theory is a match for this task. What we need is an entirely different strategy. One key to this strategy is the distinction between sets and classes: this might give us more than $\mathbf{V}$ but not more of the same.

\section{Sets and Classes}

\subsection{Sets as Classes}

Classes. A growing number of philosophers of mathematics has reminded us that two conceptions of a 'collection' have to be sharply distinguished, i.e. combinatorial collections and logical collections. This distinction finds it historical roots in the divide between the logicists (Frege, Russell, Quine) and the set-theoreticians (Cantor, Zermelo, Fraenkel, Von Neumann). ${ }^{11}$ A logical collection, or synonymously class, E say, is the extension of a predicate, $\psi(\cdot)$ say, i.e. the whole that contains exactly those things which fall under $\boldsymbol{\psi}(\cdot)$; this is expressed by Church's Schema: $X \in E \longleftrightarrow \psi(X)$. A class simply is the kind of thing which has members that pass a criterion and is completely determined by its members (Extensionality), full stop. Prior to every class is the predicate which tells us which things belong to its class-extension and which do not. The only attributes and interrelations we are permitted to ascribe to classes are those they inherit from the predicates of which they are extensions. Look at how the following class-theoretical operations mirror the logical connectives: intersection of extensions/conjunction of predicates, non-membership/negation of the predicate, union/disjunction, subset/indicative conditional, etc. The license to perform these basic operations with classes are logicist desirables if not necessities, but for other, notably Cantorian necessities, the logicist has no taste, e.g. well-foundedness, ordinal numbers, cardinal numbers and choice-sets.

Sets. A combinatorial collection, or synonymously set, is the product of combinatorial activities, as formally codified by the axioms and theorems of set-theory. "A set is the result of combining into a whole definite, distinct objects of our thought or intuition", as Cantor famously said [1895, §1]. The meaning of the psychological notion of a definite,

\footnotetext{
${ }^{11}$ Maddy [1983, pp.117-119] has traced awareness of the distinction back to the German mathematician J. König (1904), and has found it explicitly in the works of Zermelo, Gödel and Martin. We add Frege [1984, p. 212], much earlier than König, Russell [1919, pp.12-13] and Bernays [1958, p.56] to this lot. See also Lavine [1994, pp. 63-77].
} 
distinct object of thought is admittedly not as bright as the sun shining on a hot summer's day, but it has proved sufficiently illuminating for most mathematicians to give some guidance through the dark, as a star in the sky on a cold winter's night. The heuristic motivation for the axioms of ZFC usually is 'combinatorial' through-and-through: making union-sets, pair-sets, power-sets, replacement-sets, subsets and choice-sets out of given sets seems so plausible because we can somehow easily imagine these 'combinatorial activities' without having to drift on the high plains of abstraction. Eggs and apples will do. And we fiercely extrapolate from the realm of the market place to the realm of infinite sets. This combinatorial understanding of the set-concept is standardly conceived to be captured rigourously by the cumulative hierarchy of all pure sets: a set is the kind of thing that is produced by the set-cumulator $\alpha \mapsto \mathbf{\Psi}(\alpha)$.

The conceptual distinction between the combinatorial set and the logical class (where the adjectives 'combinatorial' and 'logical' are now redundant but will occasionally be added for emphasis) helps us, for example, to understand the vehement historical strife over the Axiom of Choice: it is a clash of different meanings. The proponents of Choice (Zermelo, Fraenkel) usually have the combinatorial set in mind, whereas the adversaries of Choice (Borel, Lebesgue, Russell, Quine) have the logical class in the back of their mind. Recall that Choice postulates a 'whole' without giving a predicate for it that determines it. If 'whole' means 'set', then Choice is plausible because we can so easily imagine the combinatorial activity that produces a choice-set: choosing members from every set contained in a given set is like choosing one sweet from every jar in a candy store. But if, on the other hand, 'whole' means 'class', then Choice is implausible because of the very reason that for a class we first must have a predicate of which it is the extension, we must have a criterion to decide whether something belongs to it or not; this is something Choice exactly fails to deliver - the very reason why Zermelo's Separation Schema cannot give us a choice-set. ${ }^{12}$

For the sake of simplicity, rather than using 'whole', or 'collection', as the general term to encompass both sets and classes, we twist things a little by preferring to use 'class' as the general term and to consider a set as a particular kind of class. Like a class, a set necessarily has members (save $\varnothing$ ) and is determined by its members (Extensionality). Like a class, a set can be considered as the extension of some predicate, but it need not be. Unlike a class, a set necessarily is well-founded, well-orderable, increasible and has a unique ordinal number when its members are ordered well, and a unique cardinal number. In ancient terminology, these are essential attributes of a set and accidental attributes of a class. Sets are what Cantorian set-theories are all about, e.g. ZFC and CVN; classes are what logicist theories are all about, e.g. Frege's Grundgesetze, Whitehead \& Russell's Principia Mathematica and Quine's Mathematical Logic.

\footnotetext{
${ }^{12}$ Cf. Maddy [1983, pp. 118-120], Lavine [1994, pp. 103-117]; see for Choice the Section 'Speakable and Unspeakable in Set-Theory' in Muller [1998, pp. 502-505].
} 


\subsection{Requirements for a Founding Theory}

Our founding theory will be a synthesis between Cantorian set-theory and logicist class-theory, thus 'dialectically transcending' both set-theory and class-theory to a theory of the ruling classes, intended to encompas Cantorian set-theory, category-theory and carrying an extended Bourbakian architecture of mathematics consisting of class-structures rather than set-structures. In this fashion we can exploit the similarities between sets and classes. We list six requirements for such a founding theory of mathematics.

(A) Class Reductionism. Every mathematical object is a class that demonstrably exists; and every variable used in some branch of mathematics is a variable over a fixed range, which is a class that demonstrably exists. Mathematics can be construed as pure class-theory. 
(B) Sets and Classes. The conceptual distinction between the combinatorial set and the logical class is honoured: sets are a particular type of class, which are wellfounded, increasable, well-ordered and have a unique ordinal number when ordered well and a unique cardinal number; and there are classes which are not sets.

(C) Cantor's Paradise. There exists a class of all sets that can pass as the cumulative hierarchy as we know it.

(D) Categoristan. Category-theory must be founded on our theory of sets and classes without falling prey to the objections of Superabundancy and Insufficiency.

(E) Bourbakistan. A concept of mathematical structure needs to be developed that extends Bourbaki's concept of a set-structure, and a structural architecture of the whole of mathematics needs to be erected that encompasses Bourbaki's original architecture of set-structures.

(F) Practice. Practice-hampering features are unwelcome, e.g. requiring definitions to be predicative (Russell) or stratified (Quine).

A few comments are in order. Class Reductionism (A) implies we can found categorytheory on our class-theory. When we further treat the domains of discourse of mathematical theories as mathematical objects, then they are classes by virtue of the first part of (A) - with the possible exception of the domain of discourse of the founding theory itself. The requirement (B) of honouring the distinction between sets and classes should not come as a surprise after the previous Section. Why do we desire to have classes besides sets and not some other mathematical object? One reason is thoroughly logicist: classes are logical abstract objects because of their generality, universal applicability and topic neutrality; when one has predicates that apply to whatever, it is a small step to have their extensions containing whatever, so that predication can be replaced with classmembership. Another reason is Occam's Principle of Parsimony. Since sets are a particular type of class (B), we shall arrive at a parsimonious domain of discourse for the whole of mathematics: nothing but classes exist, some of them being sets. There must also be classes which are not sets, otherwise the conceptual distinction would collapse logically. The next requirement (C), Cantor's Paradise, means that we want to encompass Cantorian set-theory and all its delights, e.g. a theory of the infinite.

Requirement (D), Categoristan, needs no further comment; see also the arguments supporting Claim I (§1.1). Part of requirement (D) follows from (A) but we have mentioned it for the sake of emphasis. Concerning (E), Bourbakistan, we mentioned earlier that category-theory is thoroughly Bourbakian in spirit. Categories, functor-categories, toposes and what have you, ought to be species of Bourbakian set-structures. But we know they cannot be. This is however not because Bourbaki's conception of structure is wrong; it is because the Bourbakian architecture of set-structures employs a language $\left(\mathcal{L}_{\epsilon}\right)$ 
which is too sparse and is erected on pillars which are too weak. The conceptual innovation of having classes as well as sets, as a particular kind of class, must carry over to a more general definition of structure (presumably just Bourbaki's set-structures but now classes replacing sets), such that we can build an architecture of class-structures that encompasses Bourbaki's original architecture of set-structures, i.e. Bourbakistan. Thus NeoBourbakistan arises. Concerning the final requirement (F) Practice: we find a founding theory unappealing if it outlaws what the working mathematician is doing, rather than validates it.

\section{A Theory of Sets and Classes}

\subsection{The Language of Sets and Classes}

Our theory of sets and classes, which formally is a slight deductive extension of Ackermann's set-theory [1956], is formulated in a 1st-order language, which we denote by $\mathcal{L}_{\mathrm{sc}}$. The deductive apparatus of $\mathcal{L}_{\mathrm{sc}}$ is the same as that of the formal language $\mathcal{L}_{\in}$ of settheory, which is 1st-order classical predicate logic or preferrably the system of 1st-order natural deducton. The primitive vocabulary of $\mathcal{L}_{\mathrm{SC}}$ is a slight amendation of $\mathcal{L}_{\epsilon}$. First of all we speak of class-variables rather than set-variables, and we have class-membership $(\in)$, rather than set-memberhsip, as the only primitive relation. Further we have one primitive class-name, $\mathbf{V}$, for the class of all sets. We call $\mathbf{V}$ the Cantor-class because it will turn out to contain exactly all Cantor-sets. Then a class is a set iff it is a member of $\mathbf{V}$. Ackermann [1956] originally had a primitive monadic predicate $\operatorname{Set}(\mathcal{X})$ for $\ulcorner\mathcal{X}$ is a set; the existence of $\mathbf{V}$ was then easily established as the class-extension of Set(.). For reasons of exposition it is however more expedient to have $\mathbf{V}$ around $a b$ ovo rather than the predicate Set $(\cdot)$.

Grewe [1969, p.484] proved that V of Ackermann's set-theory is undefinable in terms of $\in$ alone, which entails that $\mathbf{V}$ cannot be eliminated from $\mathcal{L}_{\mathrm{sc}}$ so as to be left with $\mathcal{L}_{\epsilon}$ again. Thus out of meta-mathematical necessity $\mathcal{L}_{\mathrm{SC}}$ is richer than $\mathcal{L}_{\in}$, an enrichment that merely consists of a single primitive term, the logical constant $\mathbf{V}$.

For the sake of emphasis and contrast, we use two sorts of variables: $A, B, C, D, \ldots$ for sets and $\mathcal{A}, \mathcal{B}, \mathcal{C}, \mathcal{D}, \ldots$ for classes. This convention is logically speaking redundant because every sentence $\varphi(X)$ in $\mathcal{L}_{\mathrm{sc}}$ with set-variable $X$ is a mere abbreviation of: $\ulcorner\mathcal{X} \in$ $\mathbf{V} \wedge \varphi(\mathcal{X})$. For emphasis of sethood we shall often write $\ulcorner X \in \mathbf{V}$ rather than $\ulcorner\mathcal{X} \in \mathbf{V}$.

The Hilbert-Bernays definition of identity for classes (and consequently for sets) reads that two classes are identical $(=)$ iff they share their members and are shared as members. We finally mention that all definitions of subclass, power-class $(\wp \mathcal{X})$, union-class $(\cup \mathcal{X})$, relation, category, arrow, functor, function, operation, structure, etc., carry over from $\mathcal{L}_{\epsilon}$ to $\mathcal{L}_{\mathrm{sc}}$, as well as the usual names for sets $\left(\aleph_{\alpha}, \omega\right.$, etc. $)$. 


\subsection{Axioms for Sets and Classes}

We shall denote Ackermann's [1956] axioms jointly by A', its extension with Regularity by $A^{\prime} R$, and its extension with our generalisation of one of Ackermann's axiom schemas by A; the theory we shall adopt is A extended with Regularity and Choice for sets, denoted by ARC.

Class Existence. We start with the most uncontroversial axiom in the history of settheory, which expresses that a class is determined when its members are determined.

Axiom of Extensionality (Ext). Classes are identical if they have the same members.

The converse is a theorem of logic due to the Hilbert-Bernays definition of identity; hence two classes are identical iff they are each other's subclass. If there is such a thing as an 'analytic truth' about classes, then Extensionality is the prime candidate. The next axiom permits us to make classes of sets at will.

Axiom Schema of Class Existence (ClsEx). For any predicate $\varphi(\cdot, Y)$, where $Y$ stands for any finite number of set-parameters, there exists a class which contains exactly those sets for which the predicate holds.

By virtue of Extensionality every class-extension $\mathcal{A} \subseteq \mathbf{V}$ is unique. The prime motivation for ClsEx, we submit, is that it is nothing less than the perfect embodiment of the logicist character of a class: any well-defined monadic predicate leads to the existence of a class-extension, full stop. In this sense Ackermann, by restoring the Principle of Full Comprehension almost in its full glory, unintendedly lit a candle for the logicist spirit.

Ackermann's original axiomatisation $\left(\mathrm{A}^{\prime}\right)$ does not have an existence axiom to create classes of classes, which is why little can be proved in $\mathrm{A}^{\prime}$ for classes in general. However, as we shall see shortly, the existence of classes of type $\wp^{n} \mathbf{V}$ remarkably can be proved in AR. So in contrast to $\mathrm{CVN}$, subclasses of $\mathbf{V}$ are never ultimate. Clearly the presence of such classes is not exploited to the fullest because $\mathrm{A}^{\prime}$ does not permit us to grab at them with a predicate - ClsEx allows us to grab in $\mathbf{V}$. By implication all classes of type $\wp^{n} \mathbf{V}$ and their members are lying waste in $\mathrm{A}^{\prime}$. We therefore are going to replace Ackermann's axiom schema ClsEx with a more powerful axiom which (a) permits us also to 'create' classes of (non-set) classes, and which (b) entails ClsEx so that it is a generalisation of Ackermann's axiom schema. We achieve this by adopting Zermelo's Separation Schema for classes.

Axiom Schema of Class Separation (ClsSep). For any predicate $\varphi(\cdot, \mathcal{Y})$, where $\mathcal{Y}$ stands for any finite number of class-parameters, and for every class $\mathcal{Z}$, there exists a class $\mathcal{A}$ which contains exactly those members of $\mathcal{Z}$ for which $\varphi(\cdot, \mathcal{Y})$ holds. 
Just as Zermelo's Separation Schema for sets (SetSep), a theorem schema in ZFC, which parenthetically is logicist in spirit because it postulates the existence of an extension of some given predicate, ClsSep allows us to grab only in classes whose existence is already secured: $\mathcal{A} \subseteq \mathcal{Z}$. In this fashion we live safely, because we only label in $\mathcal{L}_{\mathrm{sc}}$ by a predicate what is already there in the domain of discourse. Among the available separation-classes is $\mathbf{V}$. We recover Ackermann's ClsEx by choosing $\mathbf{V}$ for $\mathcal{Z}$ and restricting $\mathcal{Y}$ to assume values from inside $\mathbf{V}$. Zermelo's Separation Schema for sets of ZFC follows in turn from ClsEx when we add to the predicate at hand $\ulcorner\mathcal{X} \in Z$; then $Z \in \mathbf{V}$ is the separation-set.

In ARC nothing more about classes is assumed! From a logicist point of view little more can be justified than Extensionality and ClsEx. We have nevertheless mentioned that the existence of subclasses, intersections, unions, complements and power-classes can be given a logicist motivation because they reflect the logical connectives between predicates and can be used to mimic quantification over predicates. We need however not assume these classes, because their existence follows from ClsSep, as a moment's thought will reveal (substitute their definitions for $\varphi(\cdot)$ in ClsSep), provided these classes are included in some antecedently existing class. Next we turn to the sets and by implication shift from a logicist perspective back to a Cantorian one.

Sets as Sharply Delineated Wholes. Ackermann [1956] interpreted Cantor's idea about the important absolute-infinite sets (best defined as, following Von Neumann: sets equinumerous to V) in that they cannot be conceived of as 'combinatorial wholes', as asserting they are indefinite, unsharply distinguished objects of thought, rather than Cantor's "definite, distinct objects of thought"; and he interpreted identically Cantor's assertion that they cannot, in contradistinction to finite and transfinite sets, be "mathematically determined". Ackermann called these unsharply delineated wholes Gesamtheiten and followed Cantor in calling the sharply delineated wholes Mengen (Eng.: sets). We take these unsharply delineated wholes to be classes which are not sets. So here comes a heuristic principle that replaces the well-known Limitation of Size Doctrine of ZFC:

Limitation of Sharpness Doctrine (Ackermann). Exactly sets are sharply delineated classes but the existence of unsharply delineated classes must also be recognised and admitted.

The meaning of this Doctrine will be sharpened when we observe how it motivates axioms.

Completeness. Let $A$ be a 'sharply delineated' class, i.e. a set. Throwing members out of $A$ will not make it 'unsharp'. Hence subclasses of sets are sets. Next consider the members of $A$. They must be 'sharply delineated' objects otherwise we could not have combined them into a set in the first place. When these members are classes, which they are because all objects in our theory are classes, then these classes are sets because 'sharply delineated' classes are sets. Hence members of sets are sets. So the class of all sets must be complete. 
Axiom of Completeness (Compl). The class of all sets $\mathbf{V}$ is complete, i.e. all classes contained or included in sets are sets.

This Axiom thus eliminates the possibility of finding non-set classes hiding inside the Cantor-class V. The Axiom of Completeness also gives us two sufficient conditions for sethood: being a member or a subclass of a set.

Set Existence. Following Reinhardt [1970, p. 246], we now attempt to delineate more sharply the concept of a 'sharply delineated whole'; this will suggest an axiom for the existence of sets. Let us abbreviate the sentence 'class $\mathcal{X}$ is a set satisfying predicate $\psi(\cdot)^{\prime}$ :

$$
\operatorname{Set} \psi(\mathbf{V}, \mathcal{X}) \Longleftrightarrow \psi(\mathcal{X}) \wedge \mathcal{X} \in \mathbf{V}
$$

where $\mathbf{V}$ is added as an 'axiomatically fixed parameter' in the predicate for the sake of transparency (it is not a parameter in the normal sense of the word). The existence of the class-extension of this predicate is guaranteed by Ackermann's existence axiom (ClsEx). In contrast to its sharply delineated members, class $\mathbf{V}$ is not sharply delineated, for if it were, it would be a set and we would have been dead meat (Russell's antinomy). Remember Cantor saying that $\mathbf{V}$ is too big a whole to be imagined in its entirety: it is unsharply delineated, we say.

When $\mathbf{V}$ is not sharply delineated, we plausibly cannot distinguish it from other, similarly unsharply delineated classes of sets. Suppose that $\mathbf{V}^{\prime}$ is such a class. Let us further suppose, for modesty, that $\mathbf{V}^{\prime} \supset \mathbf{V}$. (To suppose that $\mathbf{V}^{\prime} \subset \mathbf{V}$ would also do; but $\mathbf{V}^{\prime} \cap \mathbf{V}=\varnothing$ would not do, because then $\mathbf{V}$ and $\mathbf{V}^{\prime}$ would not be merely 'unsharply distinguished' from each other, they would be distinguished as sharply as is possible.) We now express in the meta-language what it means for some predicate $\psi(\mathbf{V}, \cdot)$ to be delineated sharply in the presence of $\mathbf{V}$ and $\mathbf{V}^{\prime}$ : whenever it is irrelevant whether we take $\mathbf{V}$ or $\mathbf{V}^{\prime}$ as the Cantor-class:

$$
\operatorname{Sharp}\left(\mathbf{V}, \mathbf{V}^{\prime}, \psi(\cdot)\right) \Longleftrightarrow \forall \mathcal{X}\left(\operatorname{Set} \psi(\mathbf{V}, \mathcal{X}) \longleftrightarrow \operatorname{Set} \psi\left(\mathbf{V}^{\prime}, \mathcal{X}\right)\right)
$$

Ackermann's Limitation of Sharpness Doctrine is then formally expressed as follows:

$$
\operatorname{Sharp}\left(\mathbf{V}, \mathbf{V}^{\prime}, \operatorname{Set} \psi(\cdot)\right) \longrightarrow \exists S \in \mathbf{V}, \forall \mathcal{X}(\mathcal{X} \in S \longleftrightarrow \operatorname{Set} \psi(\mathbf{V}, \mathcal{X})) .
$$

Suppose $\psi(\cdot)$ is such that all classes satisfying it happen to be sets:

$$
\forall \mathcal{X}(\psi(\mathcal{X}) \longrightarrow \mathcal{X} \in \mathbf{V})
$$

Then by virtue of supposition $\mathbf{V}^{\prime} \supset \mathbf{V}$ we have that:

$$
\forall \mathcal{X}\left(\psi(\mathcal{X}) \wedge \mathcal{X} \in \mathbf{V} \longleftrightarrow \psi(\mathcal{X}) \wedge \mathcal{X} \in \mathbf{V}^{\prime}\right)
$$

which is definition (9). Hence if the only classes that satisfy $\psi(\cdot)$ are sets (11), then its extension is sharply delineated: $\psi(\cdot)$ has a set-extension! Formally:

$$
\forall \mathcal{X}(\psi(\mathcal{X}) \longrightarrow \mathcal{X} \in \mathbf{V}) \longrightarrow \exists S \in \mathbf{V}, \forall \mathcal{X}(\mathcal{X} \in S \longleftrightarrow \psi(\mathcal{X}))
$$


We now straightforwardly accept (13) as our axiom schema of set-existence, provided one thing is taken care of.

Ackermann argues that when we are hunting down an axiom that gives us sets of sets, i.e. sharply delineated classes of sharply delineated classes, then, first, the unsharply delineated $\mathbf{V}$ in which we grab must not occur in $\psi(\cdot, \mathcal{Y})$, and, secondly, the parameters in $\psi(\cdot, \mathcal{Y})$ must not assume unsharp values, i.e. they must be sets. Let us call such a predicate safe:

$\operatorname{Safe}(\psi) \Longleftrightarrow \psi$ only contains set-parameters and $\mathbf{V}$ does not occur in it.

We are now in a position to formulate

Ackermann's Axiom Schema of Set Existence (AckSet). For any safe predicate $\boldsymbol{\psi}(Y, \cdot)$, where $Y$ stands for any finite number of set-parameters, if the only classes for which it holds are sets, then these sets form a set.

Ackermann's axiom is in a slogan: Have Safe Sets. If we denote by $\mathcal{S}$ the purported class-extension $^{13}$ of some safe predicate, then AckSet says:

$\mathcal{S}$ is obtained safely $\wedge \mathcal{S} \subset \mathbf{V} \longrightarrow \mathcal{S} \in \mathbf{V}$.

In AckSet something entirely different from Zermelo's Separation (SetSep) is proposed. Instead of permitting the predicate to grab at members of a given set $Z \in \mathbf{V}$, Ackermann in a sense walked a logicist avenue by letting the predicate still make a grab inside the entire class of sets $(\mathbf{V})$ but in a slightly impeded fashion. Russell required predicates to be predicative: they are not permitted to mention sets or classes of which their extension is a member; Quine required predicates to be stratified, which is a weaker constraint. ${ }^{14}$ Many definitions in mathematics are however not predicative and not stratified; but they are all safe, because it is a simple fact of mathematical practice that in definitions the whole of all sets is never mentioned and need not be mentioned as long as one is defining some set. Standardly the quantifiers in virtually all definitions of mathematics are bound to some antecedently given set. Whereas Russell and Quine impeded the grabbing-faculties of the comprehension cat severely and whereas Zermelo simply locked the cat in the cupboard of a separation-set, Ackermann polished her nails and let her freely wander about the house. We can therefore safely conclude that to outlaw the use of unsafe predicates does not hamper the practice of mathematics - this in the light of requirement $(\mathrm{F})$ of $\S 3.2$.

This comprehension cat of Ackermann's thus wanders freely about the house. Is it safe? If the Cantor-class $\mathbf{V}$ were allowed to occur in $\psi(\mathcal{Y})$, then we could form a new

\footnotetext{
${ }^{13}$ We say 'purported', because not licensed by ClsEx.

${ }^{14}$ Fraenkel [1973, p. 38, pp. 193-200].
} 
predicate $\psi(\mathcal{Y}) \wedge \bar{Y} \in \vec{V}$, which would then have yielded a set accordingly. Then choosing for $\psi(\cdot)$ the Russell predicate 'being not self-membered' would have yielded Russell's antinomy:

$$
\mathbf{R} \in \mathbf{R} \longleftrightarrow \mathbf{R} \notin \mathbf{R}, \quad \text { where } \quad \mathbf{R} \equiv\{\mathcal{Y} \mid \mathcal{Y} \notin \mathcal{Y} \wedge \mathcal{Y} \in \mathbf{V}\} \in \mathbf{V}
$$

Similarly, it can be demonstrated that lifting the restriction on set-parameters (the other restriction to make predicates safe) also leads to Russellian calamities (see Fraenkel et al. [1973, p. 150]).

Fraenkel et al. [1973, p. ] have criticised the following aspect of AckSet in the light of Ackermann's motivation: if quantification for the parameters is restricted to $\mathbf{V}$, then why is the variable allowed to go outside $\mathbf{V}$ ? (ibid.). In other words: why do we want to make sure whether there is no class outside $\mathbf{V}$ satisfying $\psi(\cdot)$ before we license the inference to call a set the extension of $\psi(\cdot)$, whose existence as a class is already secured by virtue of ClsEx?

We propose to respond as follows. If we were to restrict the variable $\mathcal{X}$ to $\mathbf{V}$, we would be presupposing that $\mathbf{V}$ is a sharply delineated whole, so that for every value that $\mathcal{X}$ assumes we can say: it is or it is not in $\mathbf{V}$. It would not begin to make sense to consider some other $\mathbf{V}^{\prime} \supset \mathbf{V}$ as the class of all sets, as Ackermann's Doctrine invites us to do. In other words, to restrict $\mathcal{X}$ would run afoul against the Limitation of Sharpness Doctrine as we have motivated it above. We have consciously accepted this Doctrine and we shall not betray it now.

Regularity. If a class were to give rise to a Mirimanoff-chain (i.e. an infinitely descending membership-chain), we would never call it sharply delineated, for then there is no end in 'unpacking' its members to see what we have on our hands; hence for every class to be a sharply delineated whole, it must be well-founded.

Axiom of Regularity (Reg). Every non-empty set has a member disjoint with it.

We expect that, just as in ZFC, Regularity will prevent various features anathema to the combinatorial set to arise, such as self-membered sets, the Narcissus set $N=\{N\}$ (which is full of itself), Siamese twin-sets $(X \in Y$ and $Y \in X)$ and Mirimanoff-chains. ${ }^{15}$

Choice. Suppose a class, $\mathcal{X}$ say, is sharply delineated. Then it is a set. According to Completeness its members are sets and their members too. The choice class then also consists of sets. Is it really a set? Arguably it is, because it is not amplinumerous to $\mathcal{X}$.

Sharply delineated class, i.e. set $X$, arguably has a unique cardinal number, for if $X$ had no cardinal number, i.e. no definite number of members, then we can hardly call it sharply delineated. Of different sharply distinguished classes we plausibly must also be able to say how their cardinalities compare; in ZF the comparability of all cardinal numbers is equivalent to the Axiom of Choice (Hartog's Theorem). Mutatis mutandis for $\mathcal{X}$ having

\footnotetext{
${ }^{15}$ See Muller [1998, pp. 38-40] for a discussion of these sets.
} 
a unique ordinal number when ordered well: since $\mathcal{X}$ is sharply delineated, counting its members in-the-generalised-sense of well-ordering them somehow, ends at a particular ordinal number that characterises the way in which the members of $\mathcal{X}$ are ordered well, i.e. it characterises the ensuing well-ordered set.

Further, without Choice, a startling number of theorems which are considered part \& parcel of mathematics become unavailable (every vector-space has a basis, etc.), and transfinite cardinal arithmetic, the crown on Cantor's theory of infinity, becomes an impossible mess: one cannot even prove the most elementary statements in cardinal arithmetic. ${ }^{16}$

Hence there is ample Cantorian motivation to adopt Choice.

Axiom of Choice (C). For every disjointed set $X$ which does not contain the empty set $\varnothing$, there exists a non-empty choice-set, i.e. a set that contains exactly one member of the sets contained in $X$.

As with Regularity, we adopt Choice for inhabitants of the Cantor-class only, not for classes generally.

Axiomatics. Let us say succinctly what we assume in ARC in order to have an overall view. First of all we have that classes are determined by their members (Ext). We have a complete class $\mathbf{V}$ of all sets (Compl). We have an elementary axiom schema to grab in classes whose existence has been secured (ClsSep), without any syntactical restrictions whatsoever; it entails Ackermann's original license to make classes of sets only (ClsEx). We also have an axiom schema to make sets of sets in a manner which safeguards us against the known antinomies (AckSet). Notation:

$$
\begin{aligned}
& \mathrm{A}^{\prime} \Longleftrightarrow \text { Ext } \wedge \text { Compl } \wedge \text { ClsEx } \wedge \text { AckSet }, \\
& \mathrm{A} \Longleftrightarrow \text { Ext } \wedge \text { Compl } \wedge \text { ClsSep } \wedge \text { AckSet } .
\end{aligned}
$$

To repeat, $\mathrm{A}^{\prime}$ is Ackermann's original theory [1956] and A is our extension with a stronger class existence schema (we then drop the prime). Fraenkel et al. [1973, p. 150] declared about Ackermann's theory that it is "the beauty of the proofs" which makes $\mathrm{A}^{\prime}$ interesting. Finally, we have a prohibition on irregularities which are baneful for the combinatorial character of sets (Regularity), and the usual axiom that prolongues to the transfinite case a combinatorial intuition about making combinatorial sets by choosing elements from available sets (Choice). The suffix ' $R C^{\prime}$ to $A^{\prime}$ and A, to obtain $A^{\prime} R C$ and $A R C$, respectively, signal their deductive extensions with Regularity (Reg) and Choice (C). ${ }^{17}$ So our founding theory of sets and classes is:

$$
\mathrm{ARC} \Longleftrightarrow \text { Ext } \wedge \text { Compl } \wedge \text { ClsSep } \wedge \text { AckSet } \wedge \operatorname{Reg} \wedge C
$$

\footnotetext{
${ }^{16}$ Cf. Muller [1998, p. 503] and references therein for lists of theorems implied by or equivalent to Choice.

${ }^{17}$ The enrichment of Ackermann's theory $\left(\mathrm{A}^{\prime}\right)$ with Regularity is due to Lévy [1959], who added it (to obtain AR) in the interest of comparing Ackermann's axioms to ZF.
} 
What we do not have is the Power Axiom, the Union Axiom, the Replacement Axiom and some Axiom of Infinity, postulating some transfinite set. So at this point we can only hope that V is Cantor's Paradise. But as one surely will expect, this hope will be fulfilled. This is the topic of the next Section, wherein we relate Ackermann's theory and our extension to ZFC.

\subsection{Logical Respect}

The purpose of this Section is to find out how ARC relates to standard set-theory (ZFC) and how strong assumptions are needed to prove its

Known Results. A sequence of results by Ackermann [1956], Lévy [1959] and Reinhardt [1970] culminated in the surprising result that in spite of the fact that Ackermann's axiomatisation looks completely different from ZF (no axiom of infinity, no axiomatic license to make power-sets, union-sets and replacement-sets), it is nonetheless so that his original theory, when extended with Regularity to obtain AR, is conservative over, hence equiconsistent with ZF. This means that the Cantor-class $\mathbf{V}$ of ARC is identical to the domain of discourse of ZFC. Ackermann's Limitation of Sharpness Doctrine gives us a novel perspective on the very same sets of ZFC.

Novel Results. All proofs of the results we are about to mention are in Muller [1998, pp.516-526]. Theorem I: the theory of sets and classes ARC is consistent relative to the set-theory $\mathrm{ZFC}^{+}$, which is ZFC plus the first non-denumerable strongly inaccessible cardinal number $\mathfrak{i}_{1}$. Since the consistency of all extant founding set-theories, i.e. Mac Lane's ZFCU and siblings, can only be proved relative to $\mathrm{ZFC}^{++}$, whereas $\mathrm{ARC}$ can be proved consistent relative to $\mathrm{ZFC}^{+}$, we conclude that $\mathrm{ARC}$ is logically superior to the competitor set-theories (Claim VI). Grothendieck's $\mathrm{ZFC}^{\omega}$ can only be proved consistent relative to a set-theory having more inaccessible cardinal numbers than there are natural numbers.

Theorem II: the consistency of the theory of sets and classes ARC can neither be proved in $\mathrm{ZFC}$ nor in $\mathrm{A}^{\prime} \mathrm{RC}$, unless these are inconsistent. The results obtained so far are depicted schematically in Figure 1. The question whether it is excluded or not that we prove in ARC more about sets in $\mathbf{V}$ than we are able to do in $\mathrm{ZFC}$ is answered by our final Theorem III: ARC is a progressive extension of $\mathrm{ZFC}$ and hence of $\mathrm{A}^{\prime} \mathrm{RC}$.

\subsection{The Lévy-Vaught Theorem}

Since there is no axiom in $\mathrm{A}^{\prime}$ which allows one to define classes of non-set classes by means of a predicate, little can be proved in $\mathrm{A}^{\prime}$ about classes in general. ${ }^{18}$ This was the reason for us to extend Ackermann's existence axiom schema for classes to a Zermelonian class Separation Schema (ClsSep). To invoke this Schema, we need however an

\footnotetext{
${ }^{18}$ For a simple argument why in AR classes do exist which have non-set members, see Fraenkel [1973, p. 153].
} 


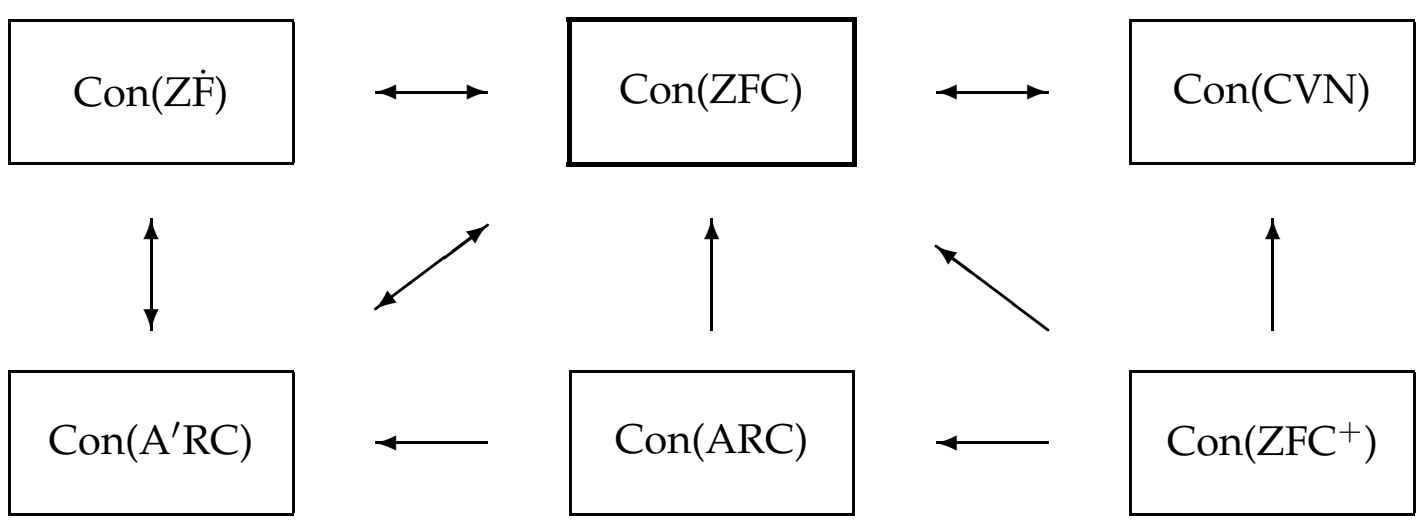

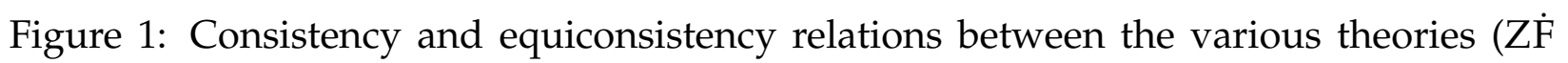
is $\mathrm{ZF}$ without Regularity). The arrow points to the weaker theory; as a consequence of Gödel's Consistency Theorem, the single arrows cannot be drawn in the opposite direction.

antecedently established separation-class and it is far from evident how to construct separation classes that go beyond $\mathbf{V}$, because the axioms of AR do not seem to provide the means to do so. In 1960 the tide turned for AR, when Lévy introduced his principles of reflection. Lévy \& Vaught [1961] proved that certain principles of reflection hold in AR, and they were able to prove the existence of classes of non-set classes of a particular type, namely classes which are in a well-defined sense 'tied to' $\mathbf{V}$. They proved that various features of sets are reflected in such 'V-tied' classes.

Earlier we defined $Y$ to be of power $n$ over $X$ iff $X \in \wp^{n} Y$. We call $\mathcal{V}$ a good class of grade $n$, for some $n \in \mathbb{N}$, iff $\mathcal{V} \in \wp^{n} \mathbf{V}$ and $n$ is its smallest power over $\mathbf{V}$. Sets are good classes of grade 0 because $\wp^{0} \mathbf{V} \equiv \mathbf{V}$. Further we can, more relevantly, define a class $\mathbf{V}$ to be good iff there is a grade such that $\mathbf{V}$ is a good class of that grade.

Theorem for Good Classes (AR). The following classes are good: ( $i$ ) the power-class and (ii) the union-class of a good class, and (iii) the union-class, the intersection-class, the complement-class, the pair-class, the ordered pair-class, and the Cartesian product-class of every $n \in \mathbb{N}^{+}$members of one good class.

In $\mathrm{A}^{\prime} \mathrm{R}$ there is no such theorem, because without ClsSep this Theorem cannot be proved. The fact that good classes are 'V-tied' implies they inherit some set-attributes, such as them being all regular. Most important and highly non-trivial is what we baptise the

Lévy-Vaught Theorem [1961, p. 1061]. All good classes exist.

Until now the Léry-Vaught Theorem was some side result without consequence for foun- 
dational investigations, laying dormant for about forty years at the periphery of metamathematical research in set-theory. We shall use it however as the Archimedean point to lift category-theory on top of the axioms of ARC. Thus only now we begin to understand the relevance of this theorem for foundational investigations. This comes about as follows.

When all classes of type $\wp^{n} \mathbf{V}$ exist, so do their members, which all are the good classes. Consequently, all classes mentioned in the Theorem above exist according to AR and ARC. An easy argument by complete induction establishes that both $\mathbf{V}$ as well every set it contains is contained and included in every $\wp^{n} \mathbf{V}$, for all $n \in \mathbb{N}$. So when we leave the Cantor-class $\mathbf{V}$ and enter the realm of good classes of positive grade, we take all sets with us in our haver sack.

It is important to realise what does and what does not follow from the Lévy-Vaught Theorem in conjunction with our Theorem for Good Classes. We can make the usual constructions of the power-, union- and pair-class, and by implication ordered pairs and Cartesian product-classes, but only with good classes of equal grade, not with a different grade $(*)$. This has not (yet) been proved. ${ }^{19}$ If we were to extend ARC with the Power and the Union Axiom for classes generally, for which there is a valid logicist motivation, we would by implication be able to make polytuples, unions and Cartesian product-classes of good classes. But then the proofs for Power and Union for sets would become redundant. Therefore we deem it better (and more challenging) not to adopt such an additional axiom for classes generally. In that case, i.e. when holding on to ARC, a simple, technical method is needed to circumvent the problem (*), which is to be found in Muller [1998, pp. 514516] (it essentially boils down to re-defining what a polytuple is). From the Lévy-Vaught Theorem we then have a theorem which is of importance for Bourbaki's conception of structure, namely a theorem that asserts the existence of arbitrary polytuples of good classes. This means we can make good polytuples and good Cartesian product-classes out of good classes of arbitrary grade; in particular we can make structure polytuples and classes of them.

\section{Conclusions and Reflections}

\subsection{Neo-Bourbakistan}

We examine whether ARC fulfils all requirements for a founding theory of mathematics we listed in advance in $\S 3.2$.

(A) Class Reductionism. When ARC is our founding theory, then every mathematical object is ultimately reduced to some class because the domain of discourse of ARC

\footnotetext{
${ }^{19}$ Such a proof was not known to Prof. A. Lévy, private E-mail communication (1997). Yours truly has failed so far to prove it. If it turns out to be provable after all, then what follows will be obsolete (all the better for us).
} 
is inhabited by classes and nothing but classes. Further, for all kinds of variables, viz. ordinal-variables, cardinal-variables, group structure-variables, relation-variables, objectand arrow-variables in a given category, etc., ZFC fails to provide a set-range over which they run. In fact the ranges needed for all kinds of variables employed by categorytheoreticians lie almost all beyond the deductive reach of $\mathrm{ZFC}$, and of $\mathrm{CVN}$ for that matter. The Lévy-Vaught Theorem and our Axiom Schema of Class Separation are together the key to found category-theory: ARC possesses ranges of most of the category-theoretical variables, as we shall argue under (D).

(B) Sets and Classes. In AR the Principle of Full Comprehension sits on the throne in the Axiom Schema of ClsEx, exactly as required by the logicist's concept of a class as the extension of a predicate. In AR we set one step forward by giving ourselves permission to grab by means of predicates where $A^{\prime} R$ was forbidden to grab: in non-set classes, without restrictions such as grabbing only predicatively. The distinction between sets and classes is honoured: for classes nothing further is assumed than that they can be extensions of predicates that apply to the members of some given class (ClsSep) and that they are determined by their members only (Extensionality). For sets a few additional axioms are assumed (Completeness, Regularity, Choice), motivated by Ackermann's Limitation of Sharpness Doctrine; these axioms guarantee that $\mathbf{V}$ comes out as the class of all and only Cantor-sets. Although all sets are classes, sets are by meta-mathematical necessity not reducible to classes, which is essentially the content of Grewe's undefinability result of $\mathbf{V}$ (mentioned in $\S 4.1$ ). This is arguably the most fascinating feature of ARC: it succeeds in having the logicist classes as the general notion and the combinatorial set as a special kind of class which one demonstrably cannot define 'away' in terms of classes. A more superior vindication of the distinction between sets and classes is hardly conceivable. It is as though the primitive concepts of a class-extension and the concomitant class-membership-relation do not suffice to found mathematics, as the logicists would have it (pace Frege and above all Russell), but that in addition some extra-logicist concept needs to be added: the good old combinatorial set (salute Cantor).

We thus conclude that the conceptual distinction between the combinatorial set and the logical class is satisfactorily codified in ARC (Claim III).

(C) Cantor's Paradise. See (B). Von Neumann's theory of ordinality and cardinality, which is the spline of the cumulative hierarchy $\Psi[\Omega]$ and the governor of the size of the sets it creates can be erected inside the confines of $\mathbf{V}$. Classes outside $\mathbf{V}$ that are not members of $\mathbf{V}$ then neither have cardinal numbers nor ordinal numbers, which is good because outside $\mathbf{V}$ we are beyond Cantorian thought and in the sphere of logicist thought. Further, $\mathbf{\Psi}$ does not rage on outside $\mathbf{V}$ so as to exhaust the entire domain of discourse of $\mathrm{ARC}$, as is the case with ZFC: it halts at the frontier of the Cantor-class.

(D) Categoristan. The definitions in $\mathcal{L}_{\epsilon}$ of arrow, category, functor and functor-category we presented in $\S 2.1$ carry over to $\mathcal{L}_{\mathrm{sc}}$ by replacing sets with classes everywhere. On the basis of the theorems of ARC we mentioned in $\S 4.4$, we see that all categories whose objects are good classes are themselves good classes, as are all ensuing functor-categories 
and all natural transformations. In this fashion every single entity studied by categorytheoreticians obtains a class-reduct. Formally speaking, the last-mentioned assertion needs to be fully proved. We have however not climbed the mountain, which is to say we have not performed the formal exercise to translated $\mathcal{L}_{\uparrow}$ into $\mathcal{L}_{\mathrm{sc}}$ and proved the axioms of category-theory formally. But we have rigourously devised a route to the top bereft of all known dangers.

Unlike all set theories with inaccessible cardinal $\mathfrak{i}_{1}$, such as all founding set-theories of $\S 2.3$, ARC does not fall prey to the objection of Insufficiency. When the categorytheoretician asks for the category Set of all sets, he obtains it with $\mathbf{V}$, because the sets living outside $\mathbf{V}$, notably in all good classes, also live inside $\mathbf{V}$. Outside $\mathbf{V}$ we generally do not find more of the same as inside $\mathbf{V}$ (sets), but a conceptually distinct entity (nonset classes); we have provided an account of their conceptual difference, we have witnessed how this difference is codified axiomatically and how the attempt to do away with the difference is a meta-mathematical impossibility according to Grewe's undefinabilitytheorem (B). Whether Lawvere's awesome category CAT of all categories is within the deductive reach of ARC is strictly speaking an open question. However, when we take 'all' to mean 'all categories studied by working mathematicians', then we can answer in the affirmative: $C A T$ exists because we can certainly separate it from a sufficiently large separation class, say $\wp^{p} \mathbf{V}$, where $p \in \mathbb{N}$ is Googolplex, say $\left(10^{100}\right.$ exponentiated to 100 again).

If the category-theoretician now suddenly wants to have some category of all classes $(\neq C l s)$, in the sense of $\mathrm{ARC}$, then we have to kiss her goodbye, for there is no such thing available in ARC. There is however no reason to desire this, because Set and $\mathrm{Cls}$ are available as the categories with 'the least structured objects', i.e. plain sets or classes of sets as objects and plain functions as arrows.

The domain of discourse of ARC is not Superabundant either, for in comparison with ZFC, which only allows for the existence of good classes of grade 0 , and with CVN, which also allows for the existence of sets of grade 1, ARC allows for classes of arbitrary grade $n \in \mathbb{N}$ according to the Lévy-Vaught Theorem, but ARC does not commit one to the gigantic domain of discourse that the inaccessibles erect - to their own destruction, by Insufficiency.

We conclude that, as promised, ARC does not fall prey to the objections of Insufficiency and Superabundance (Claim V).

(E) Bourbakistan. Bourbaki's entire theory of structure, including his seminal concept of a set-structure, carries over to ARC when we replace 'set' with 'class' everywhere. Bourbaki's Structural View of mathematics as the study of set-structures, is now adjusted to assert that mathematics is the study of class-structures. Many of these will be the familiar set-structures. The ensuing Neo-Bourbakistan is evidently more encompassing than old Bourbakistan.

According to Bourbaki there are three mother structures, which are the species of connective posets, magmas and topological spaces. They are the structures with the least 
number of 'axioms' and putatively give birth to every structure in mathematics by composing them. We propose to amend and to extend them. We list four basic species of class-structures of mathematics:

$\begin{array}{ll}\text { subset structure } & \mathfrak{S} \equiv\langle\mathcal{S}, \mathcal{F}(\mathcal{S})\rangle \\ \text { order structure } & \mathfrak{O} \equiv\langle\mathcal{V}, \triangleleft\rangle \\ \text { algebraic structure (magma) } & \mathfrak{A} \equiv\langle\mathcal{A}, \diamond\rangle \\ \text { category structure } & \mathbb{C} \equiv\langle B, \mathcal{A}(B), \circ\rangle\end{array}$

These are the four structural pillars that carry the edifice of mathematical knowledge. For the sake of generality we have replaced Bourbaki's connective poset and topological space as mother structures with the more encompassing order structure $\langle\mathcal{V}, \triangleleft\rangle$ (where $\triangleleft \in \wp \mathcal{V}^{n}$ ), and subset structure $\langle\mathcal{S}, \mathcal{F}(\mathcal{S})\rangle$ (where $\mathcal{F}(\mathcal{S}) \subseteq \wp \mathcal{S}$ ), respectively. Categorystructures are inaugurated among the new mother structures. If classes are the elementary particles of mathematics, then the mother class-structures are 'the fermions of mathematics': all mathematical 'matter' is ultimately composed of these structures, and the morphisms are 'the bosons of mathematics': they are the means by which structures interact.

Notice that we can even characterise Cantor's Paradise V within ARC, just like we can characterise any other mathematical structure, as the unique Cantor-structure:

$$
\text { Cant } \equiv\left\langle\mathbf{V}, \in_{\mathrm{v}}\right\rangle
$$

where the class-membership-relation $(\in)$ is restricted to set-membership $\left(\epsilon_{\mathrm{v}}\right)$. The Cantorstructure Cant is separated by means of ClsSep from the good class $\wp \mathbf{V} \times(\mathbf{V} \times \mathbf{V})$. ZFC is now treated on a par with every other mathematical theory: we make a grab at the class-theoretical domain of discourse of ARC by means of a class-structure predicate and obtain the subject-matter of this theory. With structure Cant (20) we haul in the cumulative hierarchy of all sets. The theory of cardinality, which has flourished so overwhelmingly in the Xxth century, resides in Cant - and could be given a more specific structural characterisation we gloss over.

(F) Practice. ARC has three comprehension schemas: one for sets of sets (AckSet), one for classes of classes (ClsSep), from which one follows for classes of sets (ClsEx). We already concluded that the mild restriction to safe predicates in AckSet does not hamper the practice of mathematics in any way; notably the usual impredicative definitions which are part \& parcel of mathematics are permitted (we emphasise that neither ClsSep nor ClsEx pose any restrictions).

We conclude that ARC satisfies our requirements for a founding theory; meeting requirements $(\mathrm{C})-(\mathrm{E})$ grounds Claim IV, and meeting requirement (E) grounds Claim V. 


\subsection{Classes and Membership: Superfluous or Unavoidable?}

There are two issues we arguably cannot evade; nonetheless we shall evade the first one but not the second. The first is the lately much discussed issue of structuralism, which is roughly the epistemic thesis that all we can possibly know is structure: the bearing of category-theory on structuralism in the context of mathematics is an issue we evade because it deserves a separate paper (cf. Muller [1998, pp. 530-550] and references therein). The second issue concerns the status of classes and sets, or both, and of the membership relation in the age of category-theory. We must and shall address it, right now.

Category-theoreticians have often expressed the desire for a theory, to quote Lawvere once more (§1.1), "in which classes and membership in classes do not play any rôle." Category-theory is supposed to be that theory. This implies that theories of sets and classes such as ARC are not wanted. Sets, classes and membership are superfluous; they even hamper the practice of category-theory. We submit two responses: (i) a defensive and (ii) an attacking one.

(i) The defensive response is that once you have presented, in Chapter 0 of your exposition of some branch of category-theory, the definitions of an arrow, a category, a functor, a topos, an allegory and what have you (§2.1), you can 'forget' about these definitions and move on. For decades this was normal practice in books and monographs when settheory was generally considered to found mathematics. There is even the often utilised possibility of not mentioning these definitions at all but only refer to where they are defined.

Even during the set-theoretical era few mathematicians have continuously thought of functions and mappings as sets of ordered pairs, but simply as rules sending one type of mathematical object to another or the same type, exactly as had been done for centuries before the advent of set-theory. But when it was now asked to them what a function is, notably whether it is necessary to have an explicit 'rule', they could or would simply present the set-theoretical definition or refer to some exposition of set-theory. Similarly, few mathematicians have continuously thought of relations and orderings as sets of order pairs, but as primitives; for every relation considered by mathematicians there was some definition available. But when it was now asked to them what a relation generally is, they could or would simply present the set-theoretical definition. And similarly for algebraic operations, equations, Etc. This author cannot think of a single reason why category-theoreticians cannot proceed similarly, gladly granted that for some branches of mathematics, such as algebraic topology and category-theory itself, $\mathcal{L}_{\uparrow}$ is a far more appropriate means of communication than bare $\mathcal{L}_{\epsilon}$. In this context Feferman [1977, p. 153] has aptly written:

I realise that workers in category-theory are so at home in their subject that they find it more natural to think in category-theoretic rather than set-theoretical terms, but I would liken this to not needing to hear once one has learned to compose music.

(ii) The attacking response is to confront the category-theoretician with his respon- 
sibilities as a founding-theoretician when he chooses to be one: to say precisely what he means and to lay all mathematical notions employed on the table. When he speaks of 'all differentiable manifolds', of 'all Abelian groups', of 'all group-homomorphisms', etc., he is undeniably speaking of wholes of some kind. The recognition of these wholes and the accompanying wholes of arrows, which together constitute a category (surely another whole of some kind), is prior to the recognition of this as a category. Feferman [1977, p.150] concludes that category-theory cannot found mathematics because it leaves the notion of a 'whole' unexamined, whereas it is evidently needed in order to understand what a category is. Feferman accepts Tarski-interpretability of all branches of mathematics in theory $\mathrm{T}$ as a necessary but not as a sufficient condition for $\mathrm{T}$ to be a founding theory for the whole of mathematics. One must add that all concepts used in every mathematical theory have counterparts in their formalised versions before being translated into the language of $T$, i.e. the formalisation must be faithful to the practice of mathematics. To pursue this argument further, when the category-theoretician says to consider some object $\mathcal{M}$ from category $M a n$ or some arrow $f$ from $\operatorname{Hom}(G r p)$, he is undeniably supposing that $\mathcal{M}$ and $f$ bear some relation to Man and $\operatorname{Hom}(\operatorname{Grp})$, respectively. And he is undeniably supposing that, conversely, Man and Hom(Grp) do not also bear that same relationship to $\mathcal{M}$ and $f$, respectively. And he is further supposing that $f$ does not bear that same relationship to itself. What is that anti-symmetric, irreflexive relation? Is it something completely different from what is usually understood by the membership-relation? Is this relation not exactly $\in$ as we know and love it? What do category-theoreticians mean when they give the following definition: "a category consists of objects and arrows such that ..."?

A theory such as ARC officially inaugurates the mentioned wholes as classes as well as the relation that $\mathcal{M}$ and $f$ bear to these classes, namely as class-membership, as well as what 'consists of' means. The battle-cry to do away with classes and the membership-relation simply makes no sense in the light of how category-theoreticians talk. The adoption of ARC means that the category-theoretician can speak forthright about classes and their members and dispense with his gaudy turning-movements.

Furthermore, it is a general principle, motivated by clarity and propounded by the likes of Cantor and Frege, that when some variable is introduced, we need to have a fixed range of the values it may assume. Now, what else can such a fixed range be but a class?

We conclude that rather than hampering the practice of mathematics, ARC founds it, the practice of category-theory included.

Acknowledgement.

I am indebted to E. van Erp for a number of valuable remarks. 


\section{References}

[Ackermann 1956] W., 'Zur Axiomatik der Mengenlehre', Mathematische Annalen 131 (1956) 336345

[Bell 1981] J.L., 'Category Theory and the Foundations of Mathematics', British Journal for the Philosophy of Science 32 (1981) 349-358

[Bell 1982] J.L., 'Categories, Toposes and Sets', Synthese 51 (1982) 293-337

[Bell 1986] J.L., 'From Absolute to Local Mathematics', Synthese 69 (1986) 409-426

[Bernays 1958] P., Axiomatic Set Theory, Amsterdam: North-Holland Publishing Company, 1958

[Bourbaki 1949] N., 'Foundations of Mathematics for the Working Mathematician', Journal of Symbolic Logic 14 (1949) 1-8

[Bourbaki 1950] N., 'The Architecture of Mathematics', American Mathematical Monthly 57 (1950) 221-232

[Cantor 1895] G., 'Beiträge zur Begründung der transfinieten Mengenlehre I', Mathematische Annalen 46 (1895) 481-512.

[Corry 1992] L., 'Nicolas Bourbaki and the Concept of Mathematical Structure', Synthese 92 (1992) $315-348$

[Feferman 1969] S., 'Set-Theoretical Foundations of Category Theory', in Mac Lane [1969, pp. 201232]

[Feferman 1977] S., 'Categorial Foundations and Foundations of Category Theory', in Logic, Foundations of Mathematics and Computability Theory, R.E. Butts \& J. Hintikka (eds.), Dordrecht: D. Reidel, 1977; pp. 149-169

[Fraenkel 1973] A.A., Y. Bar-Hillel, A. Lévy and D. van Dalen, Foundations of Set Theory (2nd Ed.), Amsterdam: North-Holland, 1973

[Frege 1983] G., Collected Papers on Mathematics, Logic and Philosophy, (ed. by B. McGuiness), Oxford: Basil Blackwell, 1983

[Goldblatt 1979] R., Topoi. The Categorial Analysis of Logic, Amsterdam: North-Holland, 1979

[Grewe 1969] R., 'Natural Models of Ackermann's Set Theory', Journal of Symbolic Logic 34 (1969) 481-488

[Joyal \& Moerdijk 1995] A. \& I., Algebraic Set Theory, Cambridge: Cambridge University Press, 1995

[Lavine 1994] S., Understanding the Infinite, Cambridge, Massachusetts: Harvard University Press, 1994 
[Lawvere 1966] F.W., 'The category of all categories as a foundation of mathematics', Proceedings of the Conference on Categorial Algebra (La Jolla, 1965), Berlin-New York: Springer-Verlag, 1966; pp. 1-21

[Lévy 1959] A., 'On Ackermann's set theory', Journal of Symbolic Logic 24 (1959) 154-166

[Lévy \& Vaught 1961] A. \& R., 'Principles of Partial Reflection in the Set Theories of Zermelo and Ackermann', Pacific Journal of Mathematics 11 (1961) 1045-1062

[Mac Lane 1969] S., (ed.), Reports of the Midwest Category Seminar III, Lecture Notes in Mathematics 106, Berlin: Springer-Verlag, 1969; 'One Universe as a Foundation for Category Theory' by Mac Lane, pp. 192-200

[Mac Lane 1986] S., Mathematics: Form and Function, Berlin: Springer-Verlag, 1986

[Mac Lane 1994] S., Categories for the Working Mathematician, Berlin: Springer-Verlag, 1994

[Mayberry 1977] J., 'On the Consistency Problem for Set Theory: An Essay on the Cantorian Foundations of Classical Mathematics', British Journal for the Philosophy of Science 28 (1977) 1-170

[Maddy 1983] P., 'Proper Classes', Journal of Symbolic Logic 48 (1983) 113-139

[McLarty 1990] C., 'The Uses and Abuses of the History of Topos Theory', British Journal for the Philosophy of Science 41 (1990) 351-375

[Muller 1998] F.A., Structures for Everyone. Contemplations and Proofs in the Foundations and Philosophy of Physics and Mathematics, Amsterdam: Gerits \& Son, 1998

[Neumann 1925] J. von, 'Eine Axiomatisierung der Mengenlehre' Journal für die Mathematik 154 (1925) 219-240, in Neumann [1961], pp. 35-47

[Neumann 1928] J. von, 'Die Axiomatisierung der Mengenlehre' Mathematisches Zeitschrift 27 (1928) 669-752, in Neumann [1961], pp. 339-422

[Neumann 1961] J. von, Collected Works I, A.H. Taub (ed.), Oxford: Pergamon Press, 1961

[Osius 1974] G., 'Categorial Set-Theory: a Characterisation of the Category of Sets', Journal for Pure and Applied Algebra 4 (1974) 79-199

[Russell 1919] B., Introduction to Mathematical Philosophy, London: Allen \& Unwin, 1919 